\title{
Tecnologia para educação em saúde na prevenção e rastreamento do câncer de mama
}

RESUMO | Objetivo: elucidar o uso de tecnologias na educação em saúde para prevenção e rastreamento do Câncer de Mama. Método: Trata-se de uma revisão integrativa realizada nas bases de dados LILACS, MEDLINE, BDENF e PUBMED com temas relacionados ao uso de tecnologia para educação em saúde na prevenção e rastreamento precoce do câncer de mama, utilizando recorte temporal de 2015 à 2019, fontes primárias e avaliação dos níveis de evidência. Resultados: Constatou-se que os estudos apontam o papel fundamental do enfermeiro frente ao educar em saúde na prevenção e rastreamento do câncer de mama, destacando o uso de tecnologias computacionais como ferramentas aliadas ao processo de empoderamento feminino e fortalecimento do seu autocuidado. Conclusão: Evidenciou-se que o uso das tecnologias em saúde é de grande valia no desenvolvimento das estratégias educativas, refletindo no fortalecimento da autonomia da mulher e melhor operacionalização destas ações nos serviços de saúde.

Palavras-chaves: Neoplasias da mama; Educação em saúde; Tecnologia biomédica; Tecnologia Educacional; Enfermagem.

\begin{abstract}
Objective: to elucidate or use of technologies in education in health for prevention and tracking of Breast Cancer. Methods: It deals with an integrative review carried out in databases LILACS, MEDLINE, BDENF and PUBMED on topics related to the use of technology for education in health in prevention and early tracking of breast cancer, using temporal cut from 2015 to 2019, primary sources and evaluation of evidence levels. Results: I confirm that the studies play the fundamental role of the nurse in the face of or educating in health in the prevention and screening of breast cancer, highlighting the use of computer technologies as allied tools to the process of feminine empowerment and strengthening of self-care. Conclusion: Evidence that I use the technologies in health and of great value, not developing educational strategies, reflecting not strengthening the autonomy of women and the operationalization of these services for health services.
\end{abstract}

Keywords: Breast neoplasms; Health education; Biomedical technology; Educational technology; Nursing.

RESUMEN | Objetivo: aclarar el uso de tecnologías en educación para la salud para la prevención y detección del cáncer de mama. Metodo: Se trata de una revisión integradora realizada en las bases de datos LILACS, MEDLINE, BDENF y PUBMED con temas relacionados con el uso de la tecnología para la educación en salud en la prevención y cribado temprano del cáncer de mama, utilizando un marco temporal de 2015 a 2019, fuentes primarias y evaluación de los niveles de evidencia. Resultados: Se encontró que los estudios señalan el papel fundamental del enfermero en la educación para la salud en la prevención y cribado del cáncer de mama, destacando el uso de tecnologías informáticas como herramientas conjugadas con el proceso de empoderamiento femenino y fortalecimiento de su autocuidado. Conclusión: Se evidenció que el uso de tecnologías en salud es de gran valor en el desarrollo de estrategias educativas, reflejando el fortalecimiento de la autonomía de las mujeres y una mejor operacionalización de estas acciones en los servicios de salud.

Palabras claves: Neoplasias de las mamas; Educación para la salud; Tecnología biomédica; Tecnologia Educacional; Enfermería.

\section{Diego Augusto Lopes Oliveira}

Enfermeiro. Doutorando em Enfermagem do PPGENF-UFPE. Docente do curso de graduação em enfermagem da ASCES-UNITA. ORCID: 0000-0003-1754-7275

\section{Carla Rayane Santos Dutra}

Enfermeira. Centro Universitário Tabosa de Almeida (ASCES-UNITA).

ORCID: 0000-0003-0321-5560

\section{Maria Eduarda Santos Silva}

Enfermeira. Centro Universitário Tabosa de Almeida (ASCES-UNITA).

ORCID: 0000-0002-4020-6981

\section{Millena Rebeca Pereira de Oliveira}

Enfermeira. Centro Universitário Tabosa de Almeida (ASCES-UNITA).

ORCID: 0000-0003-1830-9316
Luan José Queiroz de Lima

Enfermeiro. Centro Universitário Tabosa de Almeida (ASCES-UNITA).

ORCID: 0000-0001-8338-6436

\section{Alexia Silmara Pereira de Lima}

Enfermeira. Centro Universitário Tabosa de Almeida (ASCES-UNITA).

ORCID: 0000-0001-9905-7813

\section{Fernanda Portela de Carvalho}

Enfermeira. Centro Universitário Tabosa de Almeida (ASCES-UNITA).

ORCID: 0000-0001-6586-385X

Recebido em: 26/01/2021

Aprovado em: 05/02/2021
INTRODUÇÃO

videncia-se que o Câncer de
- Mama (CM) representa um pro-
blema de saúde pública, sendo a neoplasia mais incidente entre a população feminina por apresentar altas taxas de morbimortalidade nos países desenvolvidos e em desenvolvimento ${ }^{(1)}$. Identificando-se como um grupo heterogêneo de doenças, que apresenta diversas manifestações clínicas, morfológicas e genéticas que inferem nas respostas terapêuticas. Estimando-se cerca de 66.280 casos novos, para cada ano do triênio 2020-2022, conforme sua elevada magnitude, o seu controle depende essencialmente da adoção de estratégias voltadas à prevenção primária e secun- 
dária para proporcionar mudanças nesse panorama e aumentar a expectativa de vida das mulheres acometidas pela patologia ${ }^{(1-2)}$.

Acredita-se ser irrefutável que a conscientização sobre a sua gravidade é tão importante quanto a sua detecção precoce, uma vez que possibilitam a diminuição da incidência devido à redução da exposição aos fatores de risco e um prognóstico favorável para o enfrentamento da doença, no entanto percebe-se uma adesão insuficiente da população feminina aos métodos de rastreamento, bem como de informações acerca das medidas preventivas e do autocuidado, tornando-se necessária a contribuição de estratégias educativas na aquisição de conhecimentos que envolve a promoção da saúde e prevenção de complicações, que demonstra elevado potencial para modificar o comportamento dessas mulheres, por meio da conscientização e, consequentemente, diminuição dos números de casos de $\mathrm{CM} .{ }^{(3-4)}$

Entende-se frente aos aspectos supracitados que o uso das tecnologias no processo educativo em saúde tem progredido positivamente como um instrumento favorável à divulgação de informações e ao desenvolvimento da consciência crítica pelo público-alvo. Constata-se que a era da informação não deixou a área da saúde à margem, é indubitável que a inserção das tecnologias na saúde foi e é capaz de trazer os benefícios esperados para serviços de saúde e para fortalecimento do protagonismo da mulher, uma vez que o empoderamento feminino através das tecnologias educativas sobre a importância do autocuidado e desenvolvimento de hábitos que contribuem para prevenção do CM, demonstra-se a maneira mais eficaz para diminuição das elevadas taxas de morbimortalidade. ${ }^{(5-6)}$

Nota-se que o desenvolvimento de ações educativas no âmbito da saúde que abordam esse tema é uma excelente estratégia para fortalecimento e contribuição no processo de prevenção e rastreamento do $\mathrm{CM}$, sendo essencial o empenho das equipes que trabalham com a saúde da mulher na elaboração e execução dessas atividades, visando a conscientização da população, o incentivo à realização do autocuidado e o fornecimento de informações que contribuam para detecção precoce diminuindo assim os indicadores provocados pela patologia. Têm-se em vista os aspectos abordados, que tal estudo tem como objetivo, elucidar o uso de tecnologias na educação em saúde para prevenção e rastreamento do câncer de mama. ${ }^{(7)}$

\section{MÉTODO}

Trata-se de uma revisão integrativa da literatura que se caracteriza por incorporar informações e ideias que possibilitam fortalecimento da Prática Baseada em Evidências (PBE). Deu-se o processo de construção do estudo, a partir de seis etapas sistematizadas, sendo: 1 - Definição da pergunta norteadora; 2Amostragem na literatura; 3- Coleta dos dados; 4- Análise crítica dos estudos incluídos; 5- Discussão dos resultados e 6Apresentação da revisão integrativa..$^{(8-9)}$

Elencou-se enquanto pergunta norteadora: "Quais as tecnologias utilizadas para educação em saúde na prevenção e rastreamento do câncer de mama?". Desencadearam-se a partir desta as demais etapas relacionadas a seleção de materiais que possibilitaram argumentação acerca da temática baseadas no protocolo de construção de estudos de revisão PRISMA (Preferred Reporting items for Systematics Reviews and Meta-analyses).

Identificaram-se as bases de dados da LILACS, MEDLINE, BDENF e PUBMED como sítios para busca através do uso dos descritores (DECS-BIREME) ligados pelas conexões booleanas: Neoplasias da mama/ Neoplasias de la mama/ Breast neoplasms AND Educação em saúde / Health Education/ Educacion em Salud AND Tecnologia biomédica / Biomedical Technology / Tecnología biomédica OR Tecnologia Educacional / Educational Technology / Tecnología Educacional.

Consideraram-se como critérios para busca estudos de livre acesso, publicados no intervalo dos anos de 2015 a 2019, com texto na íntegra e nos idiomas português, inglês e espanhol. Eliminaram-se os estudos provenientes de trabalhos de especialização, dissertações e teses; informativos de programas de saúde específicos da área ou relacionados à temática, estudos que não apresentem relação com a temática de prevenção e rastreamento do câncer de mama e estudos de revisão integrativa da literatura. As buscas se desenvolveram nas bases de dados nos meses de março e abril de 2020.

Mediante a seleção dos artigos nas bases de dados foi estabelecido processo de análise dos dados considerando avaliação por pares ${ }^{(10)}$, através da leitura do título, do resumo e do texto completo, sendo eliminados da amostra de análise desta revisão os que não apresentassem coerência com a pergunta norteadora e objetivo de pesquisa. Utilizou-se, ainda, como método de análise, em coerência com a PBE, a classificação das publicações analisadas a partir do nível de evidência, considerando a hierarquia de evidências das pesquisas de intervenção, sendo: Nível I - revisão sistemática ou metanálise; Nível II - estudos controlados e aleatórios; Nível III - estudos controlados sem randomização; Nível IV - estudos caso-controle ou de coorte; Nível V - revisão sistemática de estudos qualitativos ou descritivos; Nível VI - estudos qualitativos ou descritivos e Nível VII - opiniões ou consensos ${ }^{(11)}$.

\section{RESULTADOS}

Utilizou-se a aplicação dos critérios estabelecidos para busca nas bases de dados. Obtendo-se 563 estudos, sendo nas bases da BVS: 380 estudos (MEDLINE: 335; LILACS: 29; BDENF: 16) e na PUBMED 183 estudos, sob os quais se 
FIGURA 1 - Fluxograma do tratamento de artigos selecionados para revisão. Caruaru-PE, 2020.

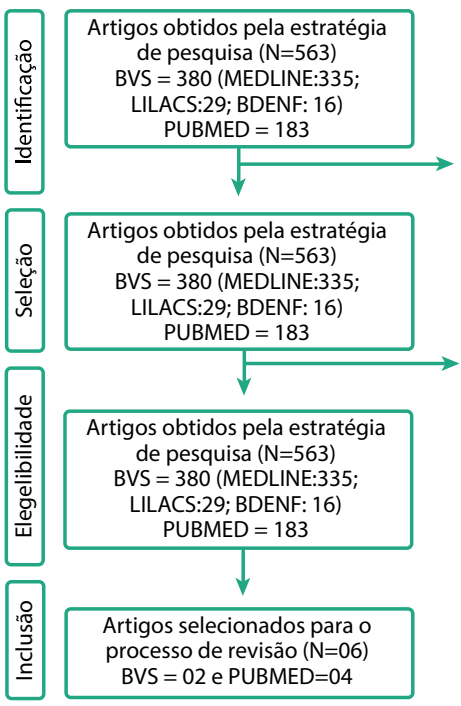

desenvolveu a etapa de identificação que deu início ao processo de análise dos dados. O detalhamento do processo de processamento dos artigos encontra-se detalhado na figura 1 :

Deu-se o processamento da amostra de artigos, mediante o processo de aplicação dos critérios de elegibilidade, o que levou a constatação dos artigos com enquadramento ao estabelecido, enquanto pergunta norteadora da revisão. Encontra-se a identificação dos estudos de forma detalhada no quadro 1 :

Realizou-se a análise de forma detalhada dos estudos incluídos para etapa final os pontos de maior relevância na produção e seu alinhamento no tocante ao direcionamento proposto pela pergunta norteadora deste estudo e formulando o detalhamento destas produções no quadro 2 :

Constatou-se frente a análise dos níveis de evidência das publicações, um vasto desenvolvimento de pesquisas de

Quadro 1- Relação dos artigos incluídos para análise após aplicação dos critérios de elegibilidade de revisão. Caruaru- PE, 2021.

\begin{tabular}{|c|c|c|c|c|}
\hline ID & TÍTULO & OBJETIVO & REVISTA/PAÍS & ANO \\
\hline 01 & $\begin{array}{l}\text { Intervención educative sobre cáncer } \\
\text { de mama en mujeres, policlínico uni- } \\
\text { versitário Emiolio Gaudinot Bueno, } \\
\text { Guatánamo 2017-2108. }{ }^{(7)}\end{array}$ & $\begin{array}{l}\text { Projetar uma intervenção educacional destinada a elevar } \\
\text { a preparação do assunto em mulheres entre } 18 \text { e } 60 \text { anos } \\
\text { de idade, na policlínica da universidade "Emílio Daudinot } \\
\text { Bueno". }\end{array}$ & $\begin{array}{l}\text { Revista información científica } \\
\text { (Cuba) }\end{array}$ & 2019 \\
\hline 02 & $\begin{array}{l}\text { Efeitos de intervenção educativa no } \\
\text { conhecimento e atitude sobre detec- } \\
\text { ção precoce do câncer de mama.(12) }\end{array}$ & $\begin{array}{l}\text { Comparar conhecimento e atitude de mulheres em relação } \\
\text { à detecção precoce do câncer de mama, antes e após aplica- } \\
\text { ção de intervenção educativa. }\end{array}$ & Revista Rene (Brasil) & 2019 \\
\hline 03 & $\begin{array}{l}\text { Harnessing Information Technology } \\
\text { to Inform Patients Facing Routine } \\
\text { Decisions: Cancer Screening as a Test } \\
\text { Case. }{ }^{(13)}\end{array}$ & $\begin{array}{l}\text { Avaliar uso de módulo de decisão e seu impacto nos cuida- } \\
\text { dos, usando três decisões de rastreamento de câncer como } \\
\text { casos de teste. }\end{array}$ & $\begin{array}{l}\text { Annals of Family Medicine } \\
\text { (Estados Unidos) }\end{array}$ & 2017 \\
\hline 04 & $\begin{array}{l}\text { Adoption, reach, and implementation } \\
\text { of a cancer education intervention in } \\
\text { African American churches. }{ }^{(14)}\end{array}$ & $\begin{array}{l}\text { Comparar a adoção, o alcance e a implementação no nível } \\
\text { organizacional e de participantes das igrejas nas quais os } \\
\text { conselheiros de saúde da comunidade de pares (ACS) foram } \\
\text { treinados usando métodos didáticos tradicionais em sala de } \\
\text { aula em comparação com um novo sistema on-line. }\end{array}$ & $\begin{array}{l}\text { Implementation Science } \\
\text { (Reino Unido) }\end{array}$ & 2017 \\
\hline 05 & $\begin{array}{l}\text { Interventions for raising breast } \\
\text { cancer awareness in women. }{ }^{(15)}\end{array}$ & $\begin{array}{l}\text { Avaliar a eficácia de intervenções para aumentar a cons- } \\
\text { cientização sobre o câncer de mama em mulheres. }\end{array}$ & $\begin{array}{l}\text { Cochrane Database of Syste- } \\
\text { matic Reviews }\end{array}$ & 2017 \\
\hline 06 & $\begin{array}{l}\text { Assessing the Effects of Participant } \\
\text { Preference and Demographics in } \\
\text { the Usage of Web-based Survey } \\
\text { Questionnaires by Women Attending } \\
\text { Screening Mammography in British } \\
\text { Columbia. }{ }^{(16)}\end{array}$ & $\begin{array}{l}\text { Investigar o impacto de fatores demográficos e preferências } \\
\text { dos participantes no uso de um questionário baseado na } \\
\text { Web em comparação com métodos mais tradicionais (cor- } \\
\text { reio e telefone) para mulheres que participam da triagem de } \\
\text { mamografia na Colúmbia Britânica, Canadá. }\end{array}$ & $\begin{array}{l}\text { Journal of Medical Internet } \\
\text { Research (Canadá) }\end{array}$ & 2016 \\
\hline
\end{tabular}


Quadro 2 - Detalhamento dos artigos incluídos para análise de texto completo. Caruaru - PE, Brasil, 2020.

\begin{tabular}{|c|c|c}
\hline ID & MÉTODO & PRINCIPAIS RESULTADOS \\
\hline 01 & $\begin{array}{c}\text { Estudo prospectivo do tipo } \\
\text { quase-experimental. O nível de } \\
\text { informação das mulheres foi } \\
\text { avaliado antes e após aplica- } \\
\text { ção da intervenção educativa, } \\
\text { classificando-as em agrupamen- } \\
\text { tos de acordo com o nível de } \\
\text { informação. }\end{array}$ & $\begin{array}{c}\text { Observou-se o aumento nas porcentagens } \\
\text { que representavam a melhoria do nível de } \\
\text { informação e melhora na preparação das } \\
\text { mulheres que se encontravam anteriormente } \\
\text { nos grupos de nível de informação insatis- } \\
\text { fatórios, revelando vantagem em realizar a } \\
\text { implementação da intervenção educativa. }\end{array}$ \\
\hline
\end{tabular}

Estudo quase-experimental, realizado com 91 mulheres através da aplicação de intervenção educativa, sendo estas divididas em quatro grupos para educação em saúde. 0 material educativo utilizado foi um folder informativo sobre detecção precoce do câncer de mama, associado à técnica de entrevista motivacional breve.

Estudo prospectivo de coorte que contou com a participação aberta de pacientes que poderiam enfrentar uma decisão de rastreamento do câncer: mulheres com idades entre 40 e 49 anos que não realizaram mamografia em dois anos. Foram excluídas participantes com diagnóstico prévio de câncer de mama ou resultados anteriores de testes de triagem anormais.

Estudo do tipo ensaio clínico randomizado (ECR) por cluster onde foram incluídas componentes de comunidades religiosas para aleatoriamente atribuir duas condições de treinamento para métodos preventivos e rastreamento do câncer de mama, sendo tradicional ou tecnologia.
A realização de programas de educação em saúde conduzidos por enfermeiros com uso dessa tecnologia e com ênfase na importância do rastreamento em mulheres assintomáticas, demonstra elevado potencial para modificar o comportamento dessas mulheres.

No primeiro ano da pesquisa, um quinto das usuárias do portal enfrentou uma potencial decisão de rastreamento do câncer. Um subconjunto considerável de pacientes e médicos relataram que o módulo ajudou as pacientes a se envolverem mais, aprimorou o conhecimento, melhorou a comunicação, tornou os clínicos mais sensíveis às preocupações dos pacientes e tornou decisões mais fáceis.
0 desenvolvimento de ações educativas no âmbito da saúde que abordem esse tema é uma excelente estratégia para fortalecimento e contribuição no processo de promoção à saúde, sendo então essencial o empenho das equipes que trabalham com a saúde da mulher na elaboração e execução dessas atividades.
NÍVEL DE EVIDÊNCIA
As intervenções voltadas para práticas preventivas e que valorizem a comunicação e informação como pontos primordiais na abordagem ao paciente devem ser realizadas, considerando aspectos culturais, cognitivos e capacidades dos envolvidos.
A implementação desse modelo de uso da tecnologia para a tomada de decisões provavelmente encontrará desafios tecnológicos de envolver as mulheres online e integrar o processo ao fluxo de trabalho prático pode não ser fácil.
III

III

IV
0 projeto teve um total de 375 participantes inscritas no projeto: 226 participantes no grupo Tradicional e 149 no grupo Tecnologia. A implementação foi forte em ambos os grupos de estudo, sendo avaliada em termos de aderência, dosagem e qualidade, sugerindo a promessa de usar métodos baseados na Web para disseminar e implementar intervenções baseadas em evidências em contextos religiosos e em outras áreas em que educadores comunitários de saúde que trabalham para eliminar as disparidades na saúde.
O Alcance no Projeto foi de 33\%, o que significa que cerca de um terço das participantes elegíveis inscritas. No trabalho relacionado na avaliação tornou-se difícil obter dados precisos sobre o número de membros por igreja, muito menos o número de membros de uma determinada faixa etária e sem um diagnóstico de câncer. Isso ilustra um desafio na obtenção de dados de alcance precisos para fins de avaliação, particularmente em pesquisa de implementação em ambientes comunitários. 


\begin{tabular}{|c|c|c|c|c|}
\hline 05 & $\begin{array}{l}\text { ECR's que avaliaram interven- } \\
\text { ções para aumentar a sensi- } \\
\text { bilização das mulheres para o } \\
\text { câncer de mama com intuito de } \\
\text { potencializar o conhecimento } \\
\text { dos sintomas/modificações } \\
\text { associadas ao câncer de mama } \\
\text { e a confiança para examinar } \\
\text { seus próprios seus seios. As } \\
\text { intervenções poderiam ser feitas } \\
\text { utilizando qualquer meio, ou } \\
\text { seja, individualmente ou em } \\
\text { grupo ou usando campanhas } \\
\text { de comunicação social voltadas } \\
\text { para populações. }\end{array}$ & $\begin{array}{l}\text { Foram incluídos dois ECR's envolvendo } 997 \\
\text { mulheres. Um ECR randomizou } 867 \text { mulheres } \\
\text { para receber um folheto escrito e cuidados } \\
\text { habituais (grupo intervenção 1), um folheto } \\
\text { escrito, cuidados habituais e uma interação } \\
\text { verbal com um radiologista ou psicólogo da } \\
\text { equipe de pesquisa (grupo de intervenção } \\
\text { 2) ou cuidados habituais (grupo controle). } 0 \\
\text { segundo ECR randomizou } 130 \text { mulheres para } \\
\text { um programa educacional (três sessões de } \\
60 \text { a } 90 \text { minutos) ou nenhuma intervenção } \\
\text { (grupo controle). As temáticas trabalhadas } \\
\text { abordaram o conhecimento dos sintomas do } \\
\text { câncer de mama, conhecimento dos riscos } \\
\text { relacionados com a idade, a frequência do } \\
\text { autoexame da mama e conscientização } \\
\text { sobre a doença. }\end{array}$ & $\begin{array}{l}\text { Com base nos resultados de dois ECR's, } \\
\text { uma breve intervenção tem o poten- } \\
\text { cial de aumentar a conscientização } \\
\text { do câncer de mama das mulheres. No } \\
\text { entanto, os resultados desta revisão } \\
\text { devem ser interpretados com cautela, } \\
\text { pois a avaliação identificou evidências } \\
\text { de qualidade moderada em apenas um } \\
\text { dos dois estudos revisados. Além disso, } \\
\text { os estudos incluídos foram heterogêneos } \\
\text { em termos de intervenções, população } \\
\text { estudada e resultados medidos. Portanto, } \\
\text { as evidências atuais não podem ser } \\
\text { generalizadas para o contexto mais } \\
\text { amplo. Estudos adicionais, incluindo } \\
\text { amostras maiores, medidas de resultados } \\
\text { validadas e abordagens longitudinais, } \\
\text { são necessários. }\end{array}$ & I \\
\hline 06 & $\begin{array}{l}\text { O estudo contou com uma } \\
\text { amostra de mulheres atendidas } \\
\text { pelo Programa Mamografia } \\
\text { Triagem de British Columbia } \\
\text { (SMPBC) que participaram de } \\
\text { um projeto de avaliação de risco } \\
\text { de câncer de mama. Agrupando } \\
\text { as participantes em quatro } \\
\text { grupos de acordo com sua faixa } \\
\text { etária: } 40-49,50-59,60-69, \text { e } \\
70 \text { a } 79 . \text { A distribuição de tipos } \\
\text { de resposta ao questionário foi } \\
\text { determinado por três fatores } \\
\text { demográficos: idade, nível } \\
\text { de instrução e etnicidade. Os } \\
\text { métodos de pesquisa analisados } \\
\text { neste estudo incluíram o uso de } \\
\text { telefone, correios e internet. }\end{array}$ & $\begin{array}{l}\text { Notou-se que participantes mais jovens } \\
\text { e aqueles com níveis mais elevados de } \\
\text { educação eram mais propensos a usar a } \\
\text { plataforma móvel; participação baseado na } \\
\text { Web não conseguiu variar entre grupos étni- } \\
\text { cos. Os resultados sugerem que é importante } \\
\text { considerar a preferência modo de pesquisa } \\
\text { do participante ao projetar e implementar } \\
\text { pesquisas desse tipo. }\end{array}$ & $\begin{array}{l}\text { A partir desse contexto, é inegável que } \\
\text { este método de pesquisa está se tornan- } \\
\text { do cada vez mais utilizados na triagem } \\
\text { do câncer da mama, através de sistemas } \\
\text { de saúde do mundo desenvolvido, } \\
\text { tornando-se imperativo que se aproxima } \\
\text { a obtenção de feedback paciente são } \\
\text { representativos da população que está } \\
\text { sendo servido. Por esta razão, clínicos } \\
\text { e investigadores deve ser diligente na } \\
\text { implementação modos de pesquisa } \\
\text { que capturam dados de seus grupos de } \\
\text { pacientes desejados. }\end{array}$ & V \\
\hline
\end{tabular}

alto poder de contribuição à PBE, reunindo informações relevantes para tomada de decisão dos profissionais de enfermagem no uso de tecnologias de educação em saúde voltadas a prevenção e rastreamento precoce do câncer de mama.

\section{DISCUSSÃo}

Constata-se, mediante a grande incidência e altas taxas de mortalidade, que o Câncer de Mama (CM) passa a ser um problema sanitário, refletindo limitações exis- tentes quanto à prevenção e realização de diagnósticos precoces, já que ensaios clínicos referem a detecção de numerosos casos em estágios mais avançados. Revela-se que este panorama retrata a deficiência no trabalho das equipes de saúde na aplicação de atividades educativas no âmbito da atenção primária referentes a prevenção dessa patologia. ${ }^{(3-7)}$

Percebe-se que o empoderamento como forma de educar e promover uma melhor tomada de decisão do grupo feminino, fortalece novos hábitos visando a prevenção do CM, sendo esta uma medida eficaz para o não surgimento ou prolongamento do caso, tendo em vista que a maioria ocorre por falta e/ou déficit de informações que as mulheres possuem. Verificou-se que o desenvolvimento de ações educativas no âmbito da saúde que abordem estratégias incisivas são essenciais, visando a prevenção e diminuição de agravos, frente a redução da exposição aos fatores de risco. ${ }^{(3-7)}$

Entende-se que no campo das práticas de saúde existem diversos modelos para re- 
alização das atividades educativas, diante do avanço tecnológico atual, o uso dessas ferramentas tem se destacado e demonstrado boa aceitação pelo público participante das atividades, devido a sua facilidade de acesso e transmissão de informações, viabilizando assim a realização da atividade educativa mediante a rotina árdua nos ambulatórios e centros de atendimento. A utilização dessas ferramentas tem se mostrado eficaz devido a sua versatilidade diante da praticidade exigida no presente, além disso as intervenções via internet, por exemplo, possibilitam uma maior facilidade de propagação, abrangendo um maior número de pessoas.

Destaca-se o profissional enfermeiro para o desenvolvimento dessas atividades, pela formação humanística e proximidade ao usuário durante a prática assistencial, contribuindo para a efetividade no processo de conscientização e conhecimento no âmbito de diversas temáticas. Insere-se no contexto da atuação da enfermagem a educação em saúde como estratégia promissora no enfrentamento de diversas problemáticas de saúde que afetam as populações em respectivos contextos sociais. ${ }^{(12)}$

Observa-se que práticas que valorizem a comunicação e informação como ponto primordial em abordagens a pacientes possuem grande impacto quando relacionadas aos aspectos culturais, cognitivos e as habilidades demonstradas por cada indivíduo, essas características incluídas em meio a entrevistas motivacionais, utilizadas em um estudo quase-experimental, demonstraram sucesso em relação ao conhecimento sobre o CM, como também na adesão e aquisição dos exames preventivos. ${ }^{(12)}$

Realizaram-se dois ensaios clínicos randomizados que utilizaram intervenções ligadas a três vertentes temáticas, sendo elas: o conhecimento da sintomatologia do CM, conhecimento dos riscos relacionados à idade e o nível de frequência de verificação da mama. Obtendo-se resultados significativos diante do uso de uma comunicação ativa e educa- tiva, desenvolvendo uma maior confiança na mulher e fortalecendo a relação interpessoal com os profissionais, conseguindo desta feita, transpor barreiras existentes quanto à procura de ajuda e proporcionando a melhoria na qualidade de vida, sendo demonstrado através do aumento da percepção das mulheres sobre as mudanças em seu corpo, como também da estima e engajamento para o autocuidado. ${ }^{(15)}$

Sustentando-se às evidências, um novo ensaio clínico reafirma o uso da interação educativa como fruto incentivador à realização das práticas supracitadas, empregando como ferramentas as tecnologias conversacionais (a Internet, mídias sociais, assistentes digitais pessoais, telefones celulares, quiosques de computador), devido o crescimento do uso de plataformas da difusão de conhecimentos, dentre os mais variados grupos de idade. ${ }^{(14)}$

Nota-se que a engenharia de informação em saúde oferece um potencial para automatizar sistematicamente os processos de tomada de decisão fora das restrições dos encontros clínicos. Possibilitando-se através de tal estratégia uma via de mão dupla, no tocante a transmissão de saber, antecipando as necessidades de decisões por meio da lógica programada, em suporte a coletar dúvidas relatadas por pacientes, além de compartilhar medidas consecutivas a próximas etapas quando e se necessárias. ${ }^{(13)}$

Sabe-se no entanto, que a utilização de abordagens alternativas para o rastreio, envolvem a tomada de decisões mais individualizadas tendo como base, fatores de risco do CM e concepções doutrinárias das mulheres, acerca da mamografia. Tornando-se sobretudo um fator inegável a afirmação de que esta estratégia tem estado cada vez mais evidente no momento de triagem do câncer, através dos sistemas de saúde. ${ }^{(16)}$

Verifica-se que a implementação da educação em saúde é vista como um fator positivo, por favorecer autonomia ao paciente, junto a atuação de enfermeiros, como educadores em saúde, capazes de utilizar de estratégias inovadoras associadas às mais diversas tecnologias. Nota-se que apesar dos benefícios advindos do uso de dispositivos tecnológicos de comunicação, clínicos e investigadores devem ser diligentes na implementação das ações educativas, sendo possível certificar que as informações transmitidas sejam fornecidas de forma simples e dinâmica à comunidade, viabilizando a compreensão do conteúdo e alcançando o objetivo proposto. Infere-se frente a essa perspectiva, a execução dessas ações quanto a mostrar seu papel, enquanto propícias na promoção de saúde e valorização do autocuidado, permitindo a elucidação e construção de novos conhecimentos no tocante às práticas de prevenção e rastreamento do $\mathrm{CM}$. $^{(3-12-16)}$

Considerou-se como limitação do estudo a quantidade de fontes atuais na literatura acerca desta temática e um grande número de publicações internacionais, demonstrando que no Brasil existe uma escassez nessa temática de pesquisa. Destaca-se a necessidade de realização de novos estudos com metodologias de maior evidência na área e que demonstrem resultados mais específicos no fortalecimento do uso das tecnologias educativas em saúde na prevenção e rastreamento precoce do $\mathrm{CM}$.

\section{CONCLUSÃO}

Conclui-se, mediante todos os aspectos abordados, que o uso das tecnologias conversacionais (internet, mídias sociais, assistentes digitais pessoais, telefones celulares, quiosques de computador) aplicadas como ferramenta educativa na propagação de informações acerca da prevenção e rastreamento do CM é uma estratégia que tem se mostrado bastante eficaz devido a sua versatilidade e funcionalismo diante da praticidade exigida na atualidade.

Nota-se que ainda há necessidade de maior discussão e avanços científicos sobre a implementação das tecnologias 
educativas em saúde e nesse sentido o papel do profissional enfermeiro é promover estratégias que valorizem a comu- nicação e informação como ponto primordial na abordagem aos pacientes, já que esse aspecto tem demonstrado muito sucesso em relação ao conhecimento sobre o CM, como também na adesão e aquisição dos exames preventivos.

\section{Referências}

1. Brasil. Ministério da Saúde. Fundação Oswaldo Cruz. Doença de Chagas. Rio de Janeiro: FIOCRUZ, 2013 [cited 2019 jun 19]. Available from: https://agencia.fiocruz. br/doen $\% C 3 \% A 7 a-d e-c h a g a s$

2. Diez M, Favaloro L, Bertolotti A, Burgos JM, Vigliano C, Lastra MP et al. Usefulness of PCR strategies for early diagnosis of Chagas' disease reactivation and treatment follow-up in heart transplantation. Am. J. Transplant. [Internet]. 2007 [cited 2020 jul 20];7(6):1633-40. doi: http://dx.doi.org/10.1111/j.1600-6143.2007.01820.x

3. Dias JCP, Ramos Junior AN, Gontijo ED, Luquetti A, Shikanai-Yasuda MA, Coura JR et al. II Consenso Brasileiro em Doença de Chagas, 2015. Epidemiol. Serv. Saúde [Internet]. 2016 [cited 2020 jun 18];25(esp):7-86. doi: http://dx.doi.org/10.5123/ S1679-49742016000500002

4. Alves DF, Muniz ASC, Abrel CDR, Freitas NR, Teixeira AB, Ferreira ES. Métodos de diagnóstico para a Doença de Chagas: uma atualização. Rev. Bras. An. Clin. [Internet]. 2018 [cited 2020 jun 18];50(4):330-3. doi: http://dx.doi.org/10.21877/24483877.201800726

5. Brasil RGA, Silva PLN, Fonseca JR, Maciel APF, Gonçalves RPF, Souto SGT et al. Análise da soroprevalência da Doença de Chagas em uma cidade do norte de Minas Gerais durante o período de 2012 a 2014. Rev. Univ. Vale Rio Verde [Internet]. 2014 [cited 2020 Jun 18];12(2):734-42. doi: http://dx.doi.org/10.5892/ruvrd.v12i2.1601

6. Forattini OP. Biogeografia, origem e distribuição da domiciliação de triatomíneos no Brasil. Rev. Saúde Pública [Internet]. 2006 [cited 2020 Jul 18];40(6):964-98. https://doi.org/10.1590/S0034-89102006000700004

7. Villar JC, Herrera VM, Carreño JGP, Herrera EV, Dominguez YZC, Vásquez SM et al. Nifurtimox versus benznidazole or placebo for asymptomatic Trypanosoma cruzi infection (Equivalence of Usual Interventions for Trypanosomiasis - EQUITY): study protocol for a randomised controlled trial. BMC [Internet]. 2019 [cited 2020 Jul 18];20(431):1-10. doi: http://dx.doi.org/ 10.1186/s13063-019-3423-3

8. Kropf SP, Azevedo N, Ferreira LO. Doença de Chagas: a construção de um fato científico e de um problema de saúde pública no Brasil. Ciênc. Saúde Colet. [Internet]. 2000 [cited 2020 Jul 18];5(2):347-65. doi: https://doi.org/10.1590/S141381232000000200009

9. Schofield CJ, Jannin J, Salvatella R. The future of Chagas disease control. Trends Parasitol. [Internet]. 2006 [cited $2020 \mathrm{Jul}$ 18];22(12):583-8. doi: http://doi.org/10.1016/j. pt.2006.09.011

10. Petherick A. Chagas disease. Nature (Lond.) [Internet]. 2010 [cited 2020 Jul 18];465(7301):10-1.

11. Coura JR, Castro SL. A critical review on Chagas disease chemotherapy. Mem. Inst. Oswaldo Cruz [Internet]. 2002 [cited 2020 Jul 19];97(1):3-24. doi: https://doi. org/10.1590/S0074-02762002000100001

12. Morilla MJ, Romero EL. Nanomedicines against Chagas disease: an update on therapeutics, prophylaxis and diagnosis. Nanomed. (Lond) [Internet]. 2015 [cited 2020 Jul 19];10(3):465-81. doi: https://doi.org/10.2217/nnm.14.185

13. Cruz CAB, SilvaALS, Alencar EMD, Santos NJB, Moreira JJS, Paixao AEA et al. Tecnologias que empregam fármacos antiparasitários para tratamento da doença Chagas. Rev. Eletr. Comun. Inf. Inov. Saúde [Internet]. 2016 [cited 2020 Jul 18];10(1):1-9: Available from: https://www.reciis.icict.fiocruz.br/index.php/reciis/article/view/1075/pdf_1075

14. Silva ACC. Investigação da atividade antiparasitária e imunomoduladora de uma nova classe de compostos tiazolidinonicos na doença de Chagas [Internet]. Recife. 90 fls. Dissertação (Mestrado em Biociências e Biotecnologia em Saúde) - Instituto Aggeu Magalhães, Fundação Oswaldo Cruz, 2018 [cited 2020 Jun 18]. Available from: https://www.arca.fiocruz.br/handle/icict/27528

15. Vinhaes MC, Dias JCP. Doença de Chagas no Brasil. Cad. Saúde Pública [Internet]. 2000 [cited 2020 May 27];16(Suppl 2):S7-S12. doi: https://doi.org/10.1590/S0102$311 \times 2000000800002$

16. Coura JR, Dias JCP. Epidemiologia, controle e vigilância da doença de Chagas: 100 anos após sua descoberta. Mem. Inst. Oswaldo Cruz [Internet]. 2009 [cited 2020 Jul 18];104(Supl. 1):31-40. doi: https://doi.org/10.1590/S0074-02762009000900006 17. Dias JCP, Vinhaes MC, Silveira AC, Schofield CJ, Cardoso B, Coura JR. Pesquisas prioritárias sobre doença de Chagas na Amazonia: agenda de curto-médio prazo Rev. Soc. Bras. Med. Trop. [Internet]. 2001 [cited 2020 Jul 18];34(5):497-8. doi: http:// doi.org/10.1590/S0037-86822001000500017

18. Victora CG, Wagstaff A, Schellenberg JA, Gwatkin D, Claeson M, Habicht J. Applying an equity lens to child health and mortality: more of the same is not enough. Lancet [Internet]. 2003 [cited 2020 Jul 18];362(9379):233-41. doi: http://doi. org/10.1016/50140-6736(03)13917-7

19. Dias JCP, Cláudio LDG, Lima MM, Albajar-Viñas P, Silva RA, Alves RV et al. Mudanças no paradigma da conduta clínica e terapêutica da doença de Chagas: avanços e perspectivas na busca da integralidade da saúde. Epidemiol. Serv. Saúde [Internet]. 2016 [cited 2020 Jul 18];25(esp):87-90. doi: http://doi.org/10.5123/S167949742016000500003

20. Lima RS, Teixeira AB, Lima VLS. Doença de Chagas: uma atualização bibliográfica. Rev. Bras. Anal. Clín. [Internet]. 2019 [cited 2020 Jul 20];51(2):103-6. doi: http://doi. org/10.21877/2448-3877.201900727

21. Lima RS, Soares MBP, Santos RR. Terapia celular na doença de Chagas. Rev. Bras. Hematol. Hemoter. [Internet]. 2009 [citado 2020 May 28];31(Supl. 1):87-92. doi: https://doi.org/10.1590/S1516-84842009005000037

22. Ramos Junior AN, Carvalho DM. Chagas' disease: past, present and future [Editorial]. Cad. Saúde Colet. [Internet]. 2009 [cited 2020 May 28];17(4):787-94. Available from: https://www.researchgate.net/publication/247768733_Editorial_-_Chagas'_ Disease_past_present_and_future

23. Brasil. Ministério da Saúde. Secretaria de Vigilância em Saúde. Doença de Chagas aguda no Brasil: série histórica de 2000 a 2013. Bol. Epidemiol. [Internet]. 2015 [cited 2020 Jun 18];46(21):1-9. Available from: http://portalarquivos.saude.gov.br/images/ pdf/2015/agosto/03/2014-020.pdf

24. Garg N, Tarleton RL. Genetic immunization elicits antigen-specific protective immune responses and decreases disease severity in Trypanosoma cruzi infection. Infect. Immun. [Internet]. 2002 [cited 2020 Jul 18];70(10):5547-55. doi: http://doi. org/10.1128/IAI.70.10.5547-5555.2002

25. Hoft DF, Eickhoff CS. Type 1 immunity provides both optimal mucosal and systemic protection against a mucosally invasive, intracellular pathogen. Infect. Immun. [Internet]. 2005 [cited 2020 Jul 18];73(8):4934-40. doi: http://doi.org/10.1128/ IAl.73.8.4934-4940.2005

26. Passos LCS, Melo RMV, Lira YM, Oliveira NFC, Trindade T, Carvalho W et al. A doença de Chagas está associada a um desfecho ruim no acompanhamento de 1 ano após a terapia de ressincronização cardíaca. Rev. Assoc. Med. Bras. [Internet]. 2019 [cited 2020 Jul 19];65(11):1391-6. doi: https://doi.org/10.1590/18069282.65.11.1391

27. Monteiro JMC, San-Martin DL, Silva BCG, Jesus PAP, Oliveira Filho J. Anticoagulação em pacientes com manifestações cardíacas da doença de Chagas e acidente vascular cerebral isquêmico cardioembólico. Arq. Neuro-Psiquiatr. [Internet]. 2018 [cited 2020 Ago 1];76(1):22-5. doi: https://doi.org/10.1590/0004-282×20170180 28. Leite ACA, Moreira MAR, Barbosa MA, Moreira Júnior $H$, Leite PCCA, Moreira JPT. Investigação clínica e manométrica em chagásicos constipados com e sem megacólon. J. Coloproctol. (Rio J.) [Internet]. 2019 [cited 2020 Ago 2];39(2):145-52. doi: https://doi.org/10.1016/j.jcol.2018.12.002

29. Rassi DC, Vieira MLC, Furtado RG, Turco FP, Melato LH, HottaVT et al. Segurança do Ecocardiograma sob Estresse com Dobutamina-Atropina em Pacientes com Doença de Chagas. Arq. Bras. Cardiol. [Internet]. 2017 [cited 2020 Ago 27];108(2):122-8. doi: https://doi.org/10.5935/abc.20170002

30. Coura JR, Borges-Pereira J. Chagas disease: What is known and what should be improved: a systemic review. Rev. Soc. Bras. Med. Trop. [Internet]. 2012 [cited 2020 Ago 27];45(3):286-96. doi: https://doi.org/10.1590/S0037-86822012000300002 31. Barbosa-Ferreira JM, Guerra JAO, Santana Filho FS, Magalhães BML, Coelho LIARC, Barbosa MGV. Acometimento cardíaco em casos de doença de Chagas aguda da Amazônia. Arq. Bras. Cardiol. [Internet]. 2010 [cited 2020 Ago 27];94(6):147-9. doi: https://doi.org/10.1590/S0066-782X2010000600023 